\title{
Non-Ordinary Primes: A Story
}

\author{
Fernando Q. Gouvêa
}

\section{CONTENTS}

1. Niebur's Formula and Its Generalizations

2. Rankin-Cohen Brackets

3. How Many Non-Ordinary Primes?

4. Computations for $k$ Divisible by 4

5. Computations for $k \equiv 2 \bmod 4$

6. Formulas

Acknowledgements

References

This research was supported in part by grant DMS-9401313

from the National Science Foundation.
A normalized modular eigenform $f$ is said to be ordinary at a prime $p$ if $p$ does not divide the $p$-th Fourier coefficient of $\mathrm{f}$. We take $\mathrm{f}$ to be a modular form of level 1 and weight $k \in\{12,16,18,20,22,26\}$ and search for primes where $f$ is not ordinary. To do this, we need an efficient way to compute the reduction modulo $p$ of the $p$-th Fourier coefficient. A convenient formula was known for $\mathrm{k}=12$; trying to understand it leads to generalized Rankin-Cohen brackets and thence to formulas that we can use to look for non-ordinary primes. We do this for $p \leq 1000000$.

In the 1980s, H. Hida introduced the notion of a $p$-ordinary modular form, and demonstrated in a series of papers [Hida 1986a; 1986b; 1989] that it played a fundamental role in the $p$-adic theory of modular forms. In this context, one usually starts with a fixed prime $p$, and chooses forms that are ordinary. It seems inevitable to reverse the question.

Suppose we start with a normalized eigenform

$$
f=q+a_{2} q^{2}+a_{3} q^{3}+\cdots+a_{n} q^{n}+\cdots
$$

of some weight and level 1. (Here, and in the remainder of this paper, we follow the usual convention of writing

$$
q=e^{2 \pi i z}
$$

where $z$ is in the complex upper halfplane.) Then we can ask for which primes $f$ is $p$-ordinary in Hida's sense. Since we are dealing with an eigenform, the answer seems simple: $f$ is $p$-ordinary if $p$ does not divide $a_{p}$. For example, consider the unique normalized eigenform of weight 12 and level 1, namely 


$$
\begin{aligned}
\Delta= & \sum_{n=1}^{\infty} \tau(n) q^{n} \\
= & q-24 q^{2}+252 q^{3}-1472 q^{4}+4830 q^{5} \\
& \quad-6048 q^{6}-16744 q^{7}+84480 q^{8}-113643 q^{9} \\
& \quad-115920 q^{10}+534612 q^{11}+\cdots,
\end{aligned}
$$

where this equation is taken as the definition of $\tau(n)$. Since $\Delta$ is an eigenform, one can see, by factoring the coefficients, that $\Delta$ is ordinary for $p=$ 11 but not for $p=2,3,5,7$. In fact, continuing the expansion, one finds that $\Delta$ seems to be ordinary for most primes, and it seems natural to ask for more information. Is it true that "most" primes will be ordinary? Can we turn this vague phrase into precise asymptotics?

These questions seem very hard to answer, so we can turn to the computer to try to obtain some numerical data. In the case of $\Delta$, some information turns out to have been in the literature long before the importance of ordinary forms was known: a 1975 paper by Niebur described an interesting formula for computing $\tau(n)$ and used it to compute $\tau(n)$ modulo $n$ for $n$ prime and less than 65064 . (See [Niebur 1975]; the formula and the results of the computation are described below.) Niebur's result is often quoted when people mention Hida's theory (for example, [Gouvêa 1992]), but few other examples are discussed.

What is special about $\Delta$ ? The crucial fact seems to be that $\Delta$ is the unique normalized cuspform of its weight and level one, i.e., that the space of cuspforms of weight 12 and level 1 is one-dimensional. Since the same is true for weights $16,18,20,22$, and 26 , there seems to be some hope of extending Niebur's formula and performing the analogous computation in those cases. This is the starting point for this paper. We find that Niebur's identity is a special case of a generalized "Rankin-Cohen bracket" construction, and we use this construction to extend Niebur's computation.

An alternative method for doing such computations is to use the Selberg trace formula (again, because the spaces are one-dimensional). We chose the method above because it allowed us to use computation modulo $n$ throughout and did not require storing a large table of precomputed values.

\section{NIEBUR'S FORMULA AND ITS GENERALIZATIONS}

Niebur's computation is based on the formula

$$
\begin{aligned}
\tau(n) & =n^{4} \sigma(n) \\
& -24 \sum_{k=1}^{n-1}\left(35 k^{4}-52 k^{3} n+18 k^{2} n^{2}\right) \sigma(k) \sigma(n-k),
\end{aligned}
$$

where $\sigma(n)$ is the sum of the divisors of $n$. This is very convenient for the computation we need to do, for two reasons. First, it only involves $\sigma(n)$, and not the more general $\sigma_{k}(n)$ (the sum of the $k$-th powers of the divisors of $n$ ), which are much larger and therefore present more of a computational challenge. Second, and more important, the formula is easily reduced modulo $n$, to get

$$
\tau(n) \equiv-840 \sum_{k=1}^{n-1} k^{4} \sigma(k) \sigma(n-k) \bmod n,
$$

which is easy to compute (for example, for small $n$ one can compute this without needing infiniteprecision arithmetic). Using this formula, Niebur checked that the only primes between 2 and 65064 for which $\Delta$ is not ordinary are $2,3,5,7$, and 2411 . (As we will remark below, small primes - here 2, 3, 5 , and 7 -are often forced to be non-ordinary by Hida's theory.)

The best way to understand Niebur's formula is to view it as a differential equation relating $\Delta$ to the Eisenstein series $E_{2}$ of weight 2, which has Fourier expansion

$$
E_{2}=1-24 \sum_{n=1}^{\infty} \sigma(n) q^{n}
$$

whre, as before, $a=e^{2 \pi i z}$. Recall that $E_{2}$ is not a modular form. As a function on the upper half- 
plane a modular form $f$ of weight $k$ (and level 1) must satisfy

$$
\begin{aligned}
& f(z+1)=f(z), \\
& f(-1 / z)=z^{k} f(z)
\end{aligned}
$$

for any $z$ in the upper half-plane. $E_{2}$ satisfies the transformation laws

$$
\begin{aligned}
& E_{2}(z+1)=E_{2}(z) \\
& E_{2}(-1 / z)=z^{2} E_{2}(z)+\frac{12 z}{2 \pi i}
\end{aligned}
$$

which make it almost, but not quite, a modular form of weight 2 .

Let $\Theta$ be the differential operator

$$
\Theta=\frac{1}{2 \pi i} \frac{d}{d z}=q \frac{d}{d q}
$$

acting on functions on the upper half-plane. To make the notation lighter, we denote the action of $\Theta$ by a prime; thus, given a function on the upper half-plane, we write

$$
f^{\prime}(z)=\Theta(f)(z)=\frac{1}{2 \pi i} \frac{d}{d z} f(z) .
$$

In terms of Fourier expansions,

$$
f=\sum_{n=0}^{\infty} a_{n} q^{n} \quad \text { implies } \quad f^{\prime}=\sum_{n=1}^{\infty} n a_{n} q^{n} .
$$

In particular,

$$
E_{2}^{\prime}=\sum_{n=1}^{\infty} n \sigma(n) q^{n},
$$

and it is easy to see that Niebur's formula is related to products of higher derivatives of $E_{2}$.

If $f$ is a modular form of weight $k$, then $f^{\prime}$ is normally not a modular form. Nevertheless, one wants to think of $f^{\prime}$ as being "almost" a modular form, of weight $k+2$. This can be made precise in various different ways. One that is particularly intriguing in this context is the fact that the Fourier expansion of $f^{\prime}$ is indeed the $q$-expansion of a $p$ adic modular form of weight $k+2$. (See [Coleman et al. 1995] for an extended discussion of this idea.)
A more elementary point is simply to note that if $f$ is a modular form of weight $k$, then

$$
f^{\prime}-\frac{k}{12} f E_{2}
$$

is a modular form of weight $k+2$. We can think of this as saying that the way $f^{\prime}$ fails to be modular compensates for the non-modularity of $E_{2}$. (This is a special case of the results in the next section; see below.)

Using this differentiation operator, Niebur's formula can be rewritten as

$$
\Delta=\frac{1}{24} E_{2}^{(4)} E_{2}+\frac{2}{3} E_{2}^{\prime \prime \prime} E_{2}^{\prime}-\frac{3}{4} E_{2}^{\prime \prime} E_{2}^{\prime \prime} .
$$

(Note that if we give the $k$-th derivative $E_{2}^{(k)}$ weight $2+2 k$, then each term in this equation is of weight 12.)

As Niebur remarks in his paper, it is easy to prove such a formula once it is written down. If $F$ denotes the right-hand side, it is clear that

$$
F(z+1)=F(z)
$$

using the transformation property of $E_{2}$ under $z \mapsto$ $-1 / z$, one checks directly that $F(-1 / z)=z^{12} F(z)$. This shows that $F$ is a multiple of $\Delta$, and then checking the first term of the Fourier expansion gives the equality. The trick, Niebur says, is to find the equation in the first place.

Given, however, programs that can do symbolic algebra, finding such identities (if any identities exist) is also easy: one finds Fourier expansions for $E_{2}$ and its derivatives, multiplies the appropriate ones to create terms of the desired weight, and then looks for a linear combination which gives the form we are looking for.

For example, let's consider weight $k=16$. There is a unique normalized eigenform $\Delta_{16}$ of weight 16 (and level 1). If a Niebur-like formula is to exist, we hope to express $\Delta_{16}$ as a linear combination of

$$
E_{2}^{(7)}, \quad E_{2}^{(6)} E_{2}, \quad E_{2}^{(5)} E_{2}^{\prime}, \quad E_{2}^{(4)} E_{2}^{\prime \prime}, \quad E_{2}^{\prime \prime \prime} E_{2}^{\prime \prime \prime}
$$

(these are actually linearly dependent, so that we can do without any one of them and still be fine; 
we will omit the first one). Solving the linear equations given by the first few terms of the Fourier expansion gives

$\Delta_{16}=-\frac{1}{24} E_{2}^{(6)} E_{2}+\frac{9}{8} E_{2}^{(5)} E_{2}^{\prime}-\frac{45}{8} E_{2}^{(4)} E_{2}^{\prime \prime}+\frac{55}{12} E_{2}^{\prime \prime \prime} E_{2}^{\prime \prime \prime}$.

Once again, this is easily checked by working out how it transforms under $z \mapsto-1 / z$. As before, one can easily translate it into a formula for the coefficients that is convenient for calculation of the Fourier coefficients.

Being successful for $k=16$, we may want to try the other weights for which the space of cusp forms is one-dimensional. For $k=18,22$, and 26, we find that there is no formula of this kind. For $k=20$, we do find a formula:

$$
\begin{aligned}
\Delta_{20}=-\frac{1}{24} E_{2}^{(8)} E_{2}+\frac{7}{4} E_{2}^{(7)} & E_{2}^{\prime}-\frac{97}{6} E_{2}^{(6)} E_{2}^{\prime \prime} \\
& +\frac{677}{12} E_{2}^{(5)} E_{2}^{\prime \prime \prime}-\frac{85}{2} E_{2}^{(4)} E_{2}^{(4)} .
\end{aligned}
$$

Finding such formulas raises more questions than it answers. Why do such formulas exist when the weight is divisible by 4 ? Why do they fail to exist for other weights? It is also natural to want to place the formulas in a wider context. (For example, could we predict the coefficients in the formulas a priori?) Finally, we would like formulas for the modular forms of weights 18,22 , and 26 .

\section{RANKIN-COHEN BRACKETS}

The best way to understand the formulas in the previous section is via the theory of Rankin-Cohen brackets, which gives a family of differential operators which map modular forms to modular forms. We follow the exposition in [Zagier 1994].

Let $f$ be a modular form of weight $k$ and level 1 . Form the power series

$$
\Phi_{f}(z, X)=\sum_{n=0}^{\infty} \frac{f^{(n)}(z)}{n !(n+k-1) !}(2 \pi i X)^{n} .
$$

Since we have $f(z+1)=f(z)$, the same is true for all higher derivatives of $f$, and hence we have
$\Phi_{f}(z+1, X)=\Phi_{f}(z, X)$. The behavior under $z \mapsto$ $-1 / z$ is more complicated: we have

$$
\Phi_{f}\left(\frac{-1}{z}, \frac{X}{z^{2}}\right)=z^{k} e^{X / z} \Phi_{f}(z, X) .
$$

Proving this boils down to proving a sequence of identities describing how the $n$-th derivative of $f$ transforms:

$$
\begin{aligned}
& \frac{f^{(n)}(-1 / z)}{n !(n+k-1) !} \\
& \quad=\sum_{m=0}^{n} \frac{z^{k+n+m}}{(2 \pi i)^{n-m}(n-m) !} \frac{f^{(m)}(z)}{m !(m+k-1) !} .
\end{aligned}
$$

These identities aren't hard to prove by induction, starting with the modular property of $f$. (See [Zagier 1994] for a different proof of the functional equation for $\Phi_{f}$.)

Now suppose we are given two modular forms $f$ and $g$ of weights $k$ and $l$, and we consider the power series

$$
\begin{aligned}
& \Phi_{f}(z, X) \Phi_{g}(z,-X) \\
&=\sum_{n=0}^{\infty} \frac{[f, g]_{n}(z)}{(n+k-1) !(n+l-1) !}(2 \pi i X)^{n} .
\end{aligned}
$$

We take this equation as the definition of the functions $[f, g]_{n}$, so that

$$
\begin{aligned}
& {[f, g]_{n}(z)} \\
& \quad=\sum_{r+s=n}(-1)^{s}\left(\begin{array}{c}
n+k-1 \\
s
\end{array}\right)\left(\begin{array}{c}
n+l-1 \\
r
\end{array}\right) f^{(r)}(z) g^{(s)}(z) .
\end{aligned}
$$

The transformation law for $\Phi_{f}$ and $\Phi_{g}$ gives

$$
\begin{aligned}
\Phi_{f}\left(-1 / z, X / z^{2}\right) \Phi_{g}\left(-1 / z,-X / z^{2}\right) & \\
= & z^{k+l} \Phi_{f}(z, X) \Phi_{g}(z,-X),
\end{aligned}
$$

since the exponential factors cancel. It follows that $[f, g]_{n}$ is a modular form of weight $k+l+2 n$; it is called the $n$-th Rankin-Cohen bracket of $f$ and $g$. (See [Zagier 1994] for a lot more information on Rankin-Cohen brackets.)

Consider the case $f=g$. Then $[f, f]_{n}$ will be a linear combinations of terms of the form $f^{(r)} f^{(s)}$, 
which is exactly the sort of thing we see in the formulas in section 1. Of course, in those formulas $f=E_{2}$, which is not modular. As Henri Cohen has pointed out to me, it turns out that one only needs a very slight modification of the RankinCohen construction to handle this case.

Let's start with the "almost modular" property of $E_{2}$ :

$$
E_{2}(-1 / z)=\frac{12 z}{2 \pi i}+z^{2} E_{2}(z) .
$$

Differentiating this - that is, applying our differential operator $(1 / 2 \pi i) d / d z$ - gives

$$
E_{2}^{\prime}(-1 / z)=\frac{12 z^{2}}{(2 \pi i)^{2}}+\frac{2 z^{3}}{2 \pi i} E_{2}(z)+z^{4} E_{2}^{\prime}(z) .
$$

By induction, we get a formula for the $n$-th derivative:

$$
\begin{aligned}
\frac{E_{2}^{(n)}(-1 / z)}{n(n+1) !}= & \frac{12 z^{n+1}}{(2 \pi i)^{n+1}(n+1) !} \\
& +\sum_{m=0}^{n} \frac{z^{2+n+m}}{(2 \pi i)^{n-m}(n-m) !} \frac{E_{2}^{(m)}(z)}{m !(m+1) !} .
\end{aligned}
$$

This is very similar to formula (2.2) above; in fact, we can fit the two formulas together with a bit of notational magic:

1. We define $(-1) !=1 / 12$.

2. If $f$ is a modular form, we define its minus-first derivative to be zero: $f^{(-1)}(z)=0$.

3. On the other hand, we set $E_{2}^{(-1)}(z)=1$.

Then the transformation law

$$
\begin{aligned}
& \frac{f^{(n)}(-1 / z)}{n !(n+k-1) !} \\
& \quad=\sum_{m=-1}^{n} \frac{z^{k+n+m}}{(2 \pi i)^{n-m}(n-m) !} \frac{f^{(m)}(z)}{m !(m+k-1) !}
\end{aligned}
$$

holds both when $f$ is a modular form of weight $k$ and when $f=E_{2}$ and $k=2$. We then modify the definition of $\Phi_{f}$ accordingly:

$$
\Phi_{f}(z, X)=\sum_{n=-1}^{\infty} \frac{f^{(n)}(z)}{n !(n+k-1) !}(2 \pi i X)^{n} .
$$

As before, it follows from (2.3) that

$$
\Phi_{f}\left(\frac{-1}{z}, \frac{X}{z^{2}}\right)=z^{k} e^{X / z} \Phi_{f}(z, X) ;
$$

this leads to an extended definition of the RankinCohen brackets:

$[f, g]_{n}(z)$

$$
=\sum_{r+s=n}(-1)^{s}\left(\begin{array}{c}
n+k-1 \\
s
\end{array}\right)\left(\begin{array}{c}
n+l-1 \\
r
\end{array}\right) f^{(r)}(z) g^{(s)}(z),
$$

where now $n \geq-2,-1 \leq r, s \leq n+1, f$ and $g$ are either modular forms of weights $k$ and $l$ or are equal to $E_{2}$ (whose weight we take to be 2), and we define

$$
\left(\begin{array}{c}
m \\
-1
\end{array}\right)=\frac{m !}{(-1) !(m+1) !}=\frac{12 m !}{(m+1) !}=\frac{12}{m+1} .
$$

As before, $[f, g]_{n}$ is a modular form of weight $k+$ $l+2 n$. (One thing we do lose is the fact that the original Rankin-Cohen brackets have integral coefficients; when $E_{2}$ is involved, we get brackets with rational coefficients. Thus, if $f$ has integral Fourier coefficients, $\left[f, E_{2}\right]_{n}$ may only have rational Fourier coefficients.)

It's a bit surprising that our formalism leads us to define a (-2)-nd and a ( -1$)$-st bracket, but note that $[f, g]_{-2}=0$ unless $f=g=E_{2}$, in which case it is a constant (which yes, is a modular form of weight $2+2-2 \cdot 2=0)$, and that

$$
[f, g]_{-1}= \begin{cases}0 & \text { if neither } f \text { nor } g \text { is } E_{2}, \\ -12 f & \text { if } f \neq E_{2} \text { and } g=E_{2}, \\ 0 & \text { if } f=g=E_{2},\end{cases}
$$

all of which are forms of the correct weight. It's also interesting to note that if $f$ is of weight $k$ we get that

$$
\left[f, E_{2}\right]_{0}=f E_{2}-\frac{12}{k} f^{\prime}
$$

is a modular form of weight $k+2$, so that we recover the fact mentioned above. (While it is well known that $f \mapsto f^{\prime}-\frac{k}{12} f E_{2}$ maps modular forms of weight $k$ to modular forms of weight $k+2$, its interpretation as a bracket of weight 0 shows it in an unusual light. When $f$ and $g$ are modular forms, 
$[f, g]_{0}=f g$ is just multiplication. Thus, the operation $f \mapsto f^{\prime}-\frac{k}{12} f E_{2}$ is seen here as a sort of "multiplication by $E_{2}$," and the appearance of $f^{\prime}$ is seen as a correction factor.)

Let's consider the case $f=g=E_{2}$. Since in general we have $[g, f]_{n}=(-1)^{n}[f, g]_{n}$, it's clear that $[f, f]_{n}=0$ if $n$ is odd, so we need only look at even values of $n$. The case $n=-2$ was discussed above.

1. $n=0$ : We get $\left[E_{2}, E_{2}\right]_{0}=E_{2}^{2}-12 E_{2}^{\prime}$; this must be a form of weight 4 ; in fact, we have $E_{2}^{2}-12 E_{2}^{\prime}=E_{4}$.

2. $n=2$ : We get $\left[E_{2}, E_{2}\right]_{2}=-6 E_{2}^{\prime \prime \prime}+6 E_{2} E_{2}^{\prime \prime}-$ $9 E_{2}^{\prime} E_{2}^{\prime}$. This is visibly a cusp form, and should be of weight 8 , and hence we conclude that

$$
-E_{2}^{\prime \prime \prime}+E_{2} E_{2}^{\prime \prime}-\frac{3}{2} E_{2}^{\prime} E_{2}^{\prime}=0,
$$

which is well-known to be true (see, for example, [van der Pol 1951]). Differentiating this identity shows that for each $k \geq 2$, the products $E_{2}^{(r)} E_{2}^{(s)}$, with $-1 \leq r \leq s$ and $r+s=k$, are linearly dependent, as we mentioned above.

3. $n=4$ : We expect a form of weight 12 , and therefore a multiple of $\Delta_{12}$. We get

$$
\begin{aligned}
-4 E_{2}^{(5)}+10 E_{2} E_{2}^{(4)}-100 E_{2}^{\prime} E_{2}^{\prime \prime \prime}+ & 100 E_{2}^{\prime \prime} E_{2}^{\prime \prime} \\
& =-144 \Delta_{12} .
\end{aligned}
$$

This looks different from Niebur's formula in section 1 , but recall that the terms are linearly dependent. In fact, differentiating the equation we get from $n=2$ twice gives

$$
-E_{2}^{(5)}+E_{2} E_{2}^{(4)}-E_{2}^{\prime} E_{3}^{\prime \prime \prime}-2 E_{2}^{\prime \prime} E_{2}^{\prime \prime}=0,
$$

and subtracting 4 times this equation from the original, then dividing by -144 , yields Niebur's formula for $\Delta_{12}$.

4. Similarly, taking $n=6$ and $n=8$ yields (formulas equivalent to) the formulas for $\Delta_{16}$ and $\Delta_{20}$ in section 1.

5. What if we take $n=10$ ? Then $\left[E_{2}, E_{2}\right]_{10}$ is a form of weight 24 , which is clearly a cusp form with rational coefficients. Of course, the eigenforms of weight 24 do not have rational coefficients, so that this is not an eigenform.

Thus we see that all the formulas in section 1 are examples of (extended) Rankin-Cohen brackets. We can also see the reason for the restriction to weights that are divisible by 4 : the bracket $\left[E_{2}, E_{2}\right]_{n}$ is of weight $4+2 n$, and it is non-zero only when $n$ is even.

If we want to get similar formulas for weight $k=18,22$, and 26 , we can use brackets $\left[E_{2}, E_{4}\right]_{n}$, which will be modular forms of weight $6+2 n$. We are most interested in what happens for $n$ even, $0 \leq n \leq 10$, which will give forms of the weights we need. (In this case we are not restricted to even $n$, but odd $n$ will give us formulas for weights 12 , 16 and 20 that, because they will involve the $\sigma_{3}(n)$ function, are significantly less useful computationally that those obtained from the $\left[E_{2}, E_{2}\right]_{n}$.)

Let's consider what happens for several values of $n$ :

1. $n=0$ : We get

$$
\left[E_{2}, E_{4}\right]_{0}=E_{2} E_{4}-3 E_{4}^{\prime}=E_{6},
$$

as discussed above.

2. $n=2,4$ : We should get cusp forms of weight 10 and 14, which must be zero. This gives two identities involving derivatives of $E_{2}$ and $E_{4}$ :

$\left[E_{2}, E_{4}\right]_{2}=3 E_{2}^{\prime \prime} E_{4}-15 E_{2}^{\prime} E_{4}^{\prime}+10 E_{2} E_{4}^{\prime \prime}-2 E_{4}^{\prime \prime \prime}=0$

and

$$
\begin{aligned}
{\left[E_{2}, E_{4}\right]_{4}=} & 35 E_{2}^{(4)} E_{4}-175 E_{2}^{\prime \prime \prime} E_{4}^{\prime}+210 E_{2}^{\prime \prime} E_{4}^{\prime \prime} \\
& \quad-70 E_{2}^{\prime} E_{4}^{\prime \prime \prime}+5 E_{2} E_{4}^{(4)}-\frac{3}{2} E_{4}^{(5)} \\
= & 0 .
\end{aligned}
$$

3. $n=3$ : We should get another formula for $\Delta_{12}$, and we do:

$$
\begin{aligned}
{\left[E_{2}, E_{4}\right]_{3}=} & 4 E_{2}^{\prime \prime \prime} E_{4}-36 E_{2}^{\prime \prime} E_{4}^{\prime}+60 E_{2}^{\prime} E_{4}^{\prime \prime} \\
& -20 E_{2} E_{4}^{\prime \prime \prime}+\frac{12}{7} E_{4}^{(4)} \\
= & -\frac{7002}{7} \Delta_{12} .
\end{aligned}
$$


4. $n=6$ : We get a multiple of the cuspform of weight 18. Indeed:

$$
\begin{aligned}
{\left[E_{2}, E_{4}\right]_{6}=8 } & E_{2}^{(6)} E_{4}-882 E_{2}^{(5)} E_{4}^{\prime}+2646 E_{2}^{(4)} E_{4}^{\prime \prime} \\
& -2940 E_{2}^{\prime \prime \prime} E_{4}^{\prime \prime \prime}+1260 E_{2}^{\prime \prime} E_{4}^{(4)} \\
& -189 E_{2}^{\prime} E_{4}^{(5)}+7 E_{2} E_{4}^{(6)}-\frac{6}{5} E_{4}^{(7)} \\
=- & 624 \Delta_{18} .
\end{aligned}
$$

5. Similarly for $n=8$ and $n=10$ :

$$
\begin{aligned}
{\left[E_{2}, E_{4}\right]_{8}=} & 165 E_{2}^{(8)} E_{4}-2970 E_{2}^{(7)} E_{4}^{\prime}+16632 E_{2}^{(6)} E_{4}^{\prime \prime} \\
& -38808 E_{2}^{(5)} E_{4}^{\prime \prime \prime}+41580 E_{2}^{(4)} E_{4}^{(4)} \\
& -20790 E_{2}^{\prime \prime \prime} E_{4}^{(5)}+4620 E_{2}^{\prime \prime} E_{4}^{(6)} \\
& -396 E_{2}^{\prime} E_{4}^{(7)}+9 E_{2} E_{4}^{(8)}-E_{4}^{(9)} \\
=- & 2040 \Delta_{22}
\end{aligned}
$$

and

$$
\begin{aligned}
{\left[E_{2}, E_{4}\right]_{10}=286 } & E_{2}^{(10)} E_{4}-7865 E_{2}^{(9)} E_{4}^{\prime}+70785 E_{2}^{(8)} E_{4}^{\prime \prime} \\
& -283140 E_{2}^{(7)} E_{4}^{\prime \prime \prime}+566280 E_{2}^{(6)} E_{4}^{(4)} \\
& -594594 E_{2}^{(5)} E_{4}^{(5)}+330330 E_{2}^{(4)} E_{4}^{(6)} \\
& -94380 E_{2}^{\prime \prime \prime} E_{4}^{(7)}+12870 E_{2}^{\prime \prime} E_{4}^{(8)} \\
& -715 E_{2}^{\prime} E_{4}^{(9)}+11 E_{2} E_{4}^{(10)}-\frac{6}{7} E_{4}^{(11)} \\
=- & \frac{31008}{7} \Delta_{26} .
\end{aligned}
$$

We can use these formulas to compute the Fourier coefficients of $\Delta_{18}, \Delta_{22}$ and $\Delta_{26}$ in terms of the divisor functions $\sigma(n)$ and $\sigma_{3}(n)$.

\section{HOW MANY NON-ORDINARY PRIMES?}

Given an eigenform $f$ of weight $k$, what is the distribution of the primes $p$ such that $f$ is not ordinary at $p$ ? Very little is known about this, but heuristic arguments suggest that non-ordinary primes should be rare.

What is known? First of all, if we relax our assumption that we are dealing with forms of level 1 and consider more general levels, we can look at the case of weight 2 . If $f$ is a cuspidal eigenform of weight 2 , level $N$, and integral coefficients, then $f$ corresponds to an elliptic curve defined over $\mathbb{Q}$, and $f$ is ordinary at $p$ if and only if $E$ has either good ordinary reduction or multiplicative reduction at $p$. Thus, (except for finitely many primes) nonordinary primes for $f$ are simply primes where $E$ has good supersingular reduction. The distribution of such primes has been studied, and partial results are known. For more information on this case, see [Elkies 1992], for example.

Going back to level $N=1$, we note that the case of Eisenstein series is trivial: if we normalize $G_{k}$ by requiring $a_{1}=1$, then $a_{p}=\sigma_{k-1}(p)=1+p^{k-1}$, which is never divisible by $p$, so that there are no non-ordinary primes.

In the case of cuspforms of level $N=1$, we have weight $k \geq 12$. There is very little that one can say in general. For $p=2$ and $p=3$, it is known, by [Hatada 1979], that there are no $p$-ordinary forms of level 1 . For $p \geq 5$, one can use Hida's theory to show that any form of level 1 will often fail to be ordinary at small enough primes. Let $S_{k}$ be the vector space of cuspforms of weight $k$, and let $S_{k}^{0}$ be the subspace spanned by the ordinary eigenforms. Let $d^{0}(k)$ be the dimension of this subspace. Then Hida proves that if $k_{1} \equiv k_{2} \bmod p-1$ and $k_{1} \geq$ $k_{2} \geq 3$, then $d^{0}\left(k_{1}\right)=d^{0}\left(k_{2}\right)$. Now, one knows that $S_{k}$ is of dimension zero for $k<12$ and for $k=14$; a fortiori we have $d^{0}(k)=0$ for such $k$. Now supppose we start with a form $f$ of weight $k \geq p+2$, and suppose $f$ is ordinary at $p$. Then $d^{0}(k)>0$. Since $k-(p-1) \geq 3$, it follows by Hida's theory that $d^{0}(k-(p-1))>0$ also. If $k-(p-1)$ is small enough (specifically, less than 12 or equal to 14$)$, this is a contradiction. Thus:

Proposition 3.1. Let $k \geq 0$ be an integer, let $p \geq 5$ be a prime, and let $k_{0}$ be the unique integer defined by the conditions $3 \leq k_{0} \leq p+1$ and $k \equiv k_{0} \bmod p-1$. If $k_{0} \leq 10$ or $k_{0}=14$, then there are no p-ordinary modular forms of weight $k$.

Of course, this proposition is only interesting when $k>p+1$, i.e., when $p<k-1$.

Given a cuspidal eigenform $f$ of weight $k>2$, let $\pi_{1}(x, f)$ denote the number of primes $p \leq x$ such that $f$ is not ordinary at $p$. It would be interesting 
to know something about the asymptotic behavior of $\pi_{1}(x, f)$ as $x \rightarrow \infty$.

It seems that nothing is known about this. For a very rough guess, we might assume that the value of $a_{p}$ modulo $p$ is random for $p \geq k-1$, i.e., that the probability that $a_{p} \equiv 0 \bmod p$ is $1 / p$. If so, one would expect that $\pi_{1}(x, f)=O(\log \log x)$. This gives a more precise form to the expectation that non-ordinary primes are very rare (once one has $p \geq k-1)$. Unfortunately, it is hard to test this guess numerically, since $\log \log x$ grows so slowly; there is no easy way to distinguish $O(\log \log x)$ from $O(1)$ computationally.

\section{COMPUTATIONS FOR $\mathrm{k}$ DIVISIBLE BY 4}

The formulas in section 1 are easily used to compute Fourier coefficients of the cuspforms of weight 12,16 , and 20 , particularly when we want to reduce modulo $n$. To uniformize the notation, we write $\Delta_{k}$, where $k \in\{12,16,18,20,22,26\}$, for the unique normalized eigenform of weight $k$ and level 1 , and we set $\Delta_{k}=\sum \tau_{k}(n) q^{n}$. The results in section 1 give us formulas for $\tau_{k}(n)$ modulo $n$ when $k=12,16$, or 20 :

$$
\begin{aligned}
& \tau_{12}(n) \equiv-840 \sum_{k=1}^{n-1} k^{4} \sigma(k) \sigma(n-k) \bmod n \\
& \tau_{16}(n) \equiv-6552 \sum_{k=1}^{n-1} k^{6} \sigma(k) \sigma(n-k) \bmod n \\
& \tau_{20}(n) \equiv-67320 \sum_{k=1}^{n-1} k^{8} \sigma(k) \sigma(n-k) \bmod n
\end{aligned}
$$

As long as $n$ is not too large, we can compute modulo $n$ without needing to use packages for infiniteprecision arithmetic. (This is probably the reason for the limit in Niebur's original computation.) If we want to handle large values of $n$ we need to use infinite-precision arithmetic; we used the GNU MP package to do this. One first computes and stores a table of $\sigma(m)$ for $m \leq 1000000$, then computes $\tau_{k}(n)$ modulo $n$. The first three tables on page 203 give the results for prime $n \leq 1000000$. Once we know which $n$ to look at, it is not too costly to compute the actual value of $\tau_{k}(n)$ using the PARI system, and to compute the precise power of $n$ which divides $\tau_{k}(n)$. These are included in the tables.

For the case of $\Delta_{12}$, one can in fact do much better: there are formulas (for example, due to Ramanujan) that allow very fast recursive computation of $\tau(n)$. According to Blair Kelly (email communication, May 10, 1996), who has used such formulas to perform the computations, there are in fact no non-ordinary primes $p \leq 7,196,993$ except for those listed in our table for $k=12$.

\section{COMPUTATIONS FOR $k \equiv 2 \bmod 4$}

The formulas in section 2 yield formulas for $\tau_{k}$ in terms of $\sigma$ and $\sigma_{3}$, which we can once again reduce modulo $n$ without problems. We get:

$$
\begin{aligned}
& \tau_{18}(n) \equiv 73920 \sum_{k=1}^{n-1} k^{6} \sigma(k) \sigma_{3}(n-k) \bmod n \\
& \tau_{22}(n) \equiv 355680 \sum_{k=1}^{n-1} k^{8} \sigma(k) \sigma_{3}(n-k) \bmod n \\
& \tau_{26}(n) \equiv 2550240 \sum_{k=1}^{n-1} k^{10} \sigma(k) \sigma_{3}(n-k) \bmod n
\end{aligned}
$$

These are harder to work with, since $\sigma_{3}(n)$ grows roughly like $n^{3}$. Once again we used the GNU MP package to handle infinite-precision arithmetic. This time, we need to compute and store the values of $\sigma(m)$ and $\sigma_{3}(m)$, and then proceed as before. The results are interesting: for $k=18,22$, and 26 , the only non-ordinary primes are the ones predicted by Proposition 3.1. The proposition predicts that

- $\Delta_{18}$ is not ordinary for $p=2,3,5,7,11,13$,

- $\Delta_{22}$ is not ordinary for $p=2,3,5,7,13,19$ (notice that for $p=11$ we have, with notations as in Proposition 3.1, $k_{0}=12$ ), and

- $\Delta_{26}$ is not ordinary for $p=2,3,5,7,11,13,17$, $19,23$. 


$$
k=12
$$

\begin{tabular}{|ccc|}
\hline$p$ & $\tau_{12}(p)$ & $\operatorname{ord}_{p}\left(\tau_{12}(p)\right)$ \\
\hline 2 & -24 & 3 \\
3 & 252 & 2 \\
5 & 4830 & 1 \\
7 & -16744 & 1 \\
2411 & 4542041100095889012 & 1 \\
\hline
\end{tabular}

$$
k=18
$$

\begin{tabular}{|ccc|}
\hline$p$ & $\tau_{18}(p)$ & $\operatorname{ord}_{p}\left(\tau_{18}(p)\right)$ \\
\hline 2 & -528 & 4 \\
3 & -4284 & 2 \\
5 & -1025850 & 2 \\
7 & 3225992 & 1 \\
11 & -753618228 & 1 \\
13 & 2541064526 & 1 \\
\hline
\end{tabular}

$$
k=22
$$

\begin{tabular}{|ccc|}
\hline$p$ & $\tau_{22}(p)$ & $\operatorname{ord}_{p}\left(\tau_{22}(p)\right)$ \\
\hline 2 & -288 & 5 \\
3 & -128844 & 3 \\
5 & 21640950 & 2 \\
7 & -768078808 & 1 \\
13 & -80621789794 & 1 \\
17 & 3052282930002 & 1 \\
19 & -7920788351740 & 1 \\
\hline
\end{tabular}

$$
k=16
$$

\begin{tabular}{|ccc|}
\hline$p$ & $\tau_{16}(p)$ & $\operatorname{ord}_{p}\left(\tau_{16}(p)\right)$ \\
\hline 2 & 216 & 3 \\
3 & -3348 & 3 \\
5 & 52110 & 1 \\
7 & 2822456 & 1 \\
11 & 20586852 & 1 \\
13 & -190073338 & 1 \\
59 & 9858856815540 & 1 \\
15271 & -8993438621168072057711693894248 & 1 \\
187441 & 72366564118086321196551211656933454802 & 1 \\
\hline
\end{tabular}

$$
k=20
$$

\begin{tabular}{|ccc|}
\hline$p$ & $\tau_{20}(p)$ & $\operatorname{ord}_{p}\left(\tau_{20}(p)\right)$ \\
\hline 2 & 456 & 3 \\
3 & 50652 & 3 \\
5 & -2377410 & 1 \\
7 & -16917544 & 2 \\
11 & -16212108 & 1 \\
13 & 50421615062 & 1 \\
17 & 225070099506 & 1 \\
3371 & -1247220165833479125222462786471468 & 1 \\
64709 & 487561492441266310170136412780007334338572670 & 1 \\
\hline
\end{tabular}

$$
k=26
$$

\begin{tabular}{|ccc|}
\hline$p$ & $\tau_{26}(p)$ & $\operatorname{ord}_{p}\left(\tau_{26}(p)\right)$ \\
\hline 2 & -48 & 4 \\
3 & -195804 & 3 \\
5 & -741989850 & 2 \\
7 & 39080597192 & 2 \\
11 & 8419515299052 & 1 \\
13 & -81651045335314 & 1 \\
17 & -2519900028948078 & 1 \\
19 & -6082056370308940 & 1 \\
23 & -94995280296320424 & 1 \\
\hline
\end{tabular}

Non-ordinary primes $p \leq 10^{6}$ for $\Delta_{k}$. 
The last three tables on page 203 give the results of the computation. In each case, we tested primes $p \leq 1000000$.

It is unclear whether one should ascribe any importance to the fact that in this case we find no "unexpected" non-ordinary primes, since this is certainly consistent with the heuristic estimate

$$
\pi_{1}(f, x)=O(\log \log x)
$$

Since the division into the two cases $k \equiv 0 \bmod 4$ and $k \equiv 2 \bmod 4$ is an artifact of our method of computation, it seems likely that the overall behavior is the same in both cases.

\section{FORMULAS}

We have given, above, the formulas for $\tau_{k}(n) \bmod$ $n$ that can be deduced from our identities. It may be of interest to record the full formulas for $\tau_{k}(n)$; this is done below. For $k$ divisible by 4 , the formulas involve only the $\sigma$ function, and are relatively simple. For $k \equiv 2 \bmod 4$, the formulas involve both $\sigma$ and $\sigma_{3}$, and are considerably more complicated.

Case $k \equiv 0 \bmod 4$. Let $m \in\{3,4,5\}$. Then

$$
\tau_{4 m}(n)=n^{2 m-2} \sigma(n)+\sum_{k=1}^{n-1} f_{m}(n, k) \sigma(k) \sigma(n-k),
$$

where

$$
\begin{aligned}
& f_{3}(n, k)=-840 k^{4}+1248 k^{3} n-432 k^{2} n^{2} \\
& f_{4}(n, k)=2640 k^{3} n^{3}-11160 k^{4} n^{2}+15048 k^{5} n-6552 k^{6}, \\
& f_{5}(n, k)=-24480 k^{4} n^{4}+130416 k^{5} n^{3}-253680 k^{6} n^{2}+215040 k^{7} n-67320 k^{8} .
\end{aligned}
$$

Case $k \equiv 2 \bmod 4$.

$$
\begin{gathered}
13 \tau_{18}(n)=42 n^{6} \sigma(n)-\left(35 n^{6}-6 n^{7}\right) \sigma_{3}(n)+\sum_{k=1}^{n-1} g_{18}(n, k) \sigma(k) \sigma_{3}(n-k), \\
-2040 \tau_{22}(n)=-3960 n^{8} \sigma(n)+\left(-240 n^{9}+2160 n^{8}\right) \sigma_{3}(n)+\sum_{k=1}^{n-1} g_{22}(n, k) \sigma(k) \sigma_{3}(n-k), \\
-31008 \tau_{26}(n)=-48048 n^{10} \sigma(n)+\left(18480 n^{10}-1440 n^{11}\right) \sigma_{3}(n)+\sum_{k=1}^{n-1} g_{26}(n, k) \sigma(k) \sigma_{3}(n-k),
\end{gathered}
$$

where

$$
\begin{array}{rl}
g_{18}(n, k)=8 & 40 n^{6}-27720 k n^{5}+277200 k^{2} n^{4}-1201200 k^{3} n^{3} \\
& +2522520 k^{4} n^{2}-2522520 k^{5} n+960960 k^{6} \\
g_{22}(n, k)=- & 51840 n^{8}+2695680 k n^{7}-44029440 k^{2} n^{6}+330220800 k^{3} n^{5}-1320883200 k^{4} n^{4} \\
& +2994001920 k^{5} n^{3}-3849431040 k^{6} n^{2}+2612113920 k^{7} n-725587200 k^{8} \\
g_{26}(n, k)=- & 443520 n^{10}+33264000 k n^{9}-798336000 k^{2} n^{8}+9047808000 k^{3} n^{7} \\
& -57001190400 k^{4} n^{6}+216604523520 k^{5} n^{5}-515725056000 k^{6} n^{4} \\
+ & 773587584000 k^{7} n^{3}-709121952000 k^{8} n^{2}+362440108800 k^{9} n-79077841920 k^{10} .
\end{array}
$$

Formulas for $\tau_{k}(n)$. 


\section{ACKNOWLEDGEMENTS}

A portion of this paper was presented at the conference in honor of Oliver Atkin held in Chicago in September, 1995. The resulting comments proved extremely helpful. I am particularly thankful to Henri Cohen for a number of crucial ideas and to Barry Mazur for his comments.

\section{REFERENCES}

[Coleman et al. 1995] R. F. Coleman, F. Q. Gouvêa, and N. Jochnowitz, " $E_{2}, \Theta$, and overconvergence", Internat. Math. Res. Notices 1 (1995), 23-41.

[Elkies 1992] N. D. Elkies, "Distribution of supersingular primes", pp. 127-132 in Journées arithmètiques (Luminy, 1989), Astérisque 198-200, 1992.

[Gouvêa 1992] F. Q. Gouvêa, "On the ordinary Hecke algebra", J. Number Theory 41 (1992), 178-198.

[Hatada 1979] K. Hatada, "Eigenvalues of Hecke operators on $\mathrm{SL}_{2}(\mathbb{Z})$ ", Math. Ann. 239 (1979), 75-96.
[Hida 1986a] H. Hida, "Galois representations into $\mathrm{GL}_{2}\left(\mathbb{Z}_{p}[[X]]\right)$ attached to ordinary cusp forms", Inv. Math. 85 (1986), 545-613.

[Hida 1986b] H. Hida, "Iwasawa modules attached to congruences of cusp forms", Ann. Sci. École Norm. Sup. (4) 19 (1986), 231-273.

[Hida 1989] H. Hida, "Theory of $p$-adic Hecke algebras and Galois representations", Sugaku Expositions 2 (1989), 75-102.

[Niebur 1975] D. Niebur, "A formula for Ramanujan's $\tau$-function", Ill. J. Math. 19 (1975), 448-449.

[van der Pol 1951] B. van der Pol, "On a nonlinear differential equation satisfied by the logarithm of the Jacobian theta functions, with arithmetical applications, I, II", Indagationes Math. 13 (1951), 261-271, 272-284.

[Zagier 1994] D. B. Zagier, "Modular forms and differential operators", Proc. Indian Acad. Sci. Math. Sci. 104:1 (1994), 57-75.

Fernando Q. Gouvêa, Department of Mathematics and Computer Science, Colby College, Waterville, ME 04901, USA (fqgouvea@colby.edu)

Received October 21, 1996; accepted December 21, 1996 